\title{
Petrie polygons, Fibonacci sequences and Farey maps
}

\author{
David Singerman, James Strudwick*
}

Received 5 June 2015, accepted 21 September 2015, published online 5 February 2016

\begin{abstract}
We consider the regular triangular maps corresponding to the principal congruence subgroups $\Gamma(n)$ of the classical modular group. We relate the sizes of the Petrie polygons on these maps to the periods of reduced Fibonacci sequences.
\end{abstract}

Keywords: Regular map, Petrie polygon, Fibonacci sequence.

Math. Subj. Class.: 05C10, 11B39, $20 \mathrm{HO5}$

\section{Introduction}

An interesting number theoretic problem is to determine the period of the Fibonacci sequence $\bmod n$. Here we look at the period $\sigma(n)$ of the Fibonacci sequence $\bmod n$ up to sign. A Petrie polygon on a regular map is a zig-zag path through the map and an important invariant of a regular map is the length of a Petrie polygon. The maps we consider here are those that arise out of principal congruence subgroups $\Gamma(n)$ of the classical modular group $\Gamma$. In this case It is shown that these lengths are equal to $\sigma(n)$. A particularly nice example is when $n=7$. Here the regular map is the famous map on the Klein quartic and we find $\sigma(7)=8$ giving the title "The Eightfold Way" to the sculpture by Helaman Ferguson that represents Klein's Riemann surface of genus 3 derived from the Klein quartic. This is described in the book "The eightfold way: the beauty of Klein's quartic curve", a collection of papers related to the Klein quartic edited by Silvio Levy [5].

Let $X$ be a compact orientable surface. By a map (or clean dessin d'enfant) on $X$ we mean an embedding of a graph $\mathcal{G}$ into $X$ such that $X \backslash \mathcal{G}$ is a union of simply-connected polygonal regions, called faces. A map thus has vertices, edges and faces. A directed edge is called a dart and a map is called regular if its automorphism group acts transitively on its darts. The platonic solids are the most well-known examples of regular maps. These are the regular maps on the Riemann sphere. We recall how we study maps using triangle groups. The universal map of type $(m, n)$ is the tessellation of one of the three simply connected

* Department of Mathematical studies, University of Southampton, Southampton SO17 1EH, United Kingdom E-mail addresses: ds@maths.soton.ac.uk (David Singerman), jes3g10@ soton.ac.uk (James Strudwick) 
Riemann surfaces, that is the Riemann sphere $\Sigma$, the Euclidean plane $\mathbb{C}$, or the hyperbolic plane, $\mathbb{H}$ (depending on whether the genus of $X$ is 0,1 , or $>1$ ) by regular $m$-gons with $n$ meeting at each vertex. This map is denoted by $\hat{\mathcal{M}}(m, n)$. The automorphism group, and also the conformal automorphism group, of $\hat{\mathcal{M}}(m, n)$ is the triangle group $\Gamma[2, m, n]$. In general, a map is of type $(m, n)$ if $m$ is the least common multiple of the face sizes and $n$ is the least common multiple of the vertex valencies. As shown in [3] every map of type $(m, n)$ is a quotient of $\hat{\mathcal{M}}(m, n)$ by a subgroup $M$ of the triangle group $\Gamma[2, m, n]$. Then $M$ is called a map subgroup of $\hat{\mathcal{M}}(m, n)$ or sometimes a fundamental group of $\hat{\mathcal{M}}(m, n)$, inside $\Gamma[2, m, n]$. A platonic surface is one that underlies a regular map. The map is regular if and only if $M$ is a normal subgroup of $\Gamma[2, m, n]$. Thus a platonic surface is one of the form $\mathbb{U} / M$ where $M$ is a normal subgroup of a triangle group and $\mathbb{U}$ is a simply connected Riemann surface.

It is permissible to let $m$ or $n$, or both to be $\infty$. In this paper we are particularly interested in the case where $m=3, n=\infty$. This means that the corresponding maps are triangular though in general we are not concerned with the vertex valencies. However, if the map is regular then we must have all vertex valencies equal. For example, the icosahedron is a triangular map with all vertices of valency 5 .

To study triangular maps we use the triangle group $[2,3, \infty]$ which is known to be the modular group $\Gamma=\operatorname{PSL}(2, \mathbb{Z})$ one of the most significant groups in mathematics. The regular maps correspond to normal subgroups of $\Gamma$. The most well-known normal subgroups of $\Gamma$ are the principal congruence subgroups $\Gamma(n)$ defined in section 5 . We let $\mathcal{M}_{3}(n)=\hat{\mathcal{M}}_{3}(3, \infty) / \Gamma(n)$. We call these maps principal congruence maps or PC maps.

For low values of $n$ these maps are well-known. For $n=2,3,4,5$ we get the triangle, tetrahedron, octahedron and icosahedron respectively. These are the only PC maps of genus 0 . For $n=6$ we get the regular map $\{3,6\}_{2.2}$ on the torus and for $n=7$ we get the Klein map on Klein's Riemann surface of genus 3. (See $[2,1]$ ).

\section{Petrie polygons}

A Petrie polygon in a map $\mathcal{M}$ is defined as a zig-zag path in the map. More precisely, we start at a vertex, then go along an edge to an adjacent vertex, the turn left and go to the next vertex and then turn right, etc., (or interchange left and right.) We have a path in which two consecutive edges belong to the same face but no three consecutive edges belong to the same face, [1, p. 54]. Eventually, in a finite regular map, we will come back to the original vertex.This path is called a Petrie path or Petrie polygon. The number of edges of this Petrie polygon is called the Petrie length of the map.

We now relate the Petrie polygons to triangle groups. From the triangle group $\Gamma[2, m, n]$, we can form the extended triangle group $\Gamma(2, m, n)$ which is the group generated by the reflections $R_{1}, R_{2}, R_{3}$ in the edges of a triangle with angles $\pi / 2, \pi / m, \pi / n$ where we choose our ordering so that $\Gamma(2, m, n)$ has a presentation

$$
\left\langle R_{1}, R_{2}, R_{3} \mid R_{1}^{2}=R_{2}^{2}=R_{3}^{2}=\left(R_{1} R_{2}\right)^{2}=\left(R_{2} R_{3}\right)^{m}=\left(R_{3} R_{1}\right)^{n}=1\right\rangle .
$$

If we let $X=R_{1} R_{2}, Y=R_{2} R_{3}, Z=R_{3} R_{1}$, then we find that $\Gamma[2, m, n]$ has a presentation

$$
\left\langle X, Y, Z \mid X^{2}=Y^{m}=Z^{n}=X Y Z=1\right\rangle .
$$

In section 5.2 of [1, p. 54], it is shown that $R_{1} R_{2} R_{3}$ is a transformation that goes one step around a Petrie polygon. Now 


$$
\left(R_{1} R_{2} R_{3}\right)^{2}=R_{1} R_{2} R_{3} R_{1} R_{2} R_{3}=R_{1} R_{2} R_{3} R_{2} R_{2} R_{1} R_{2} R_{3}=X Y^{-1} X^{-1} Y
$$

showing that Petrie length is twice the order of this commutator which implies that the Petrie length is independent of the Petrie polygon chosen; it is just a property of the map.

\section{The Farey map}

This is basically the map $\hat{\mathcal{M}}(3, \infty)$, which we abbreviate to $\mathcal{M}_{3}$. We construct it as follows. The vertices are the extended rationals $\mathbb{Q} \cup\{\infty\}$ and two rationals $\frac{a}{b}$ and $\frac{c}{d}$ are joined by an edge if and only if $a d-b c= \pm 1$.

This map has the following properties.

(a) There is a triangle with vertices $\frac{1}{0}, \frac{1}{1}, \frac{0}{1}$ called the principal triangle.

(b) The modular group $\Gamma$ acts as a group of automorphisms of $\mathcal{M}_{3}$.

(c) The general triangle has vertices $\frac{a}{c}, \frac{a+b}{c+d}, \frac{b}{d}$.

Thus the Farey map (Figure 1) is a triangular map with triangular faces given by (c). In [7] it is shown that this is the universal triangular map in the sense that any other triangular map on an orientable surface is a quotient of $\mathcal{M}_{3}$ by a subgroup $\Lambda$ of the modular group $\Gamma$. As $\mathcal{M}_{3}$ has vertices the extended rationals this means that every triangular map the vertices can be given coordinates which are $\Lambda$ orbits of points in $\mathbb{Q} \cup\{\infty\}$. We shall denote the orbit of $\frac{a}{b}$ by $\left[\frac{a}{b}\right]$. This is illustrated in [2] where there are many examples, in particular coordinates for the triangular platonic solids are given. Also see Figure 2.

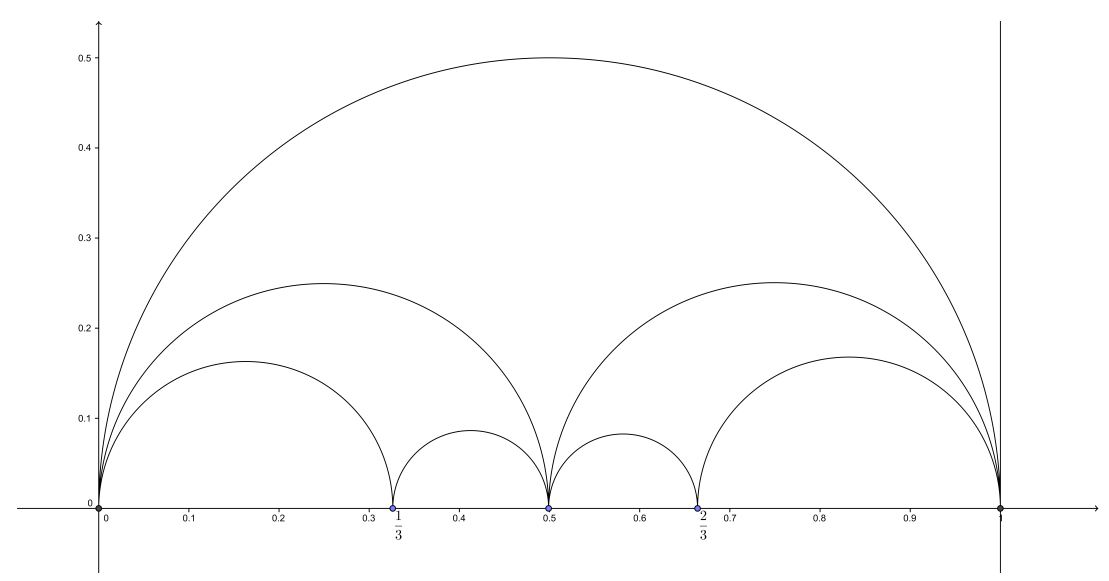

Figure 1: Farey map

\section{The Petrie polygons of the Farey map}

We consider a Petrie path in $\mathcal{M}_{3}$. By transitivity we may assume it's first edge goes from $W_{1}=\frac{1}{0}$ to $W_{2}=\frac{0}{1}$. A left turn then takes us to $W_{3}=\frac{1}{1}$ Now a right turn takes us to $W_{4}=\frac{1}{2}$. By applying a modular transformation $\left(\begin{array}{ll}a & b \\ c & d\end{array}\right)$ to the vertices $\infty, 0$ and 1 to the 
principal triangle we find that three consecutive vertices of the Petrie polygon are $\frac{a}{c}, \frac{b}{d}, \frac{a+b}{c+d}$, that is the third vertex is the Farey median of the previous two. As the first two vertices of the Petrie polygon are $\frac{1}{0}$ and $\frac{1}{1}$ the $k$ th vertex of the Petrie polygon is equal to $\frac{f_{k-1}}{f_{k}}$ where $f_{k}$ is the $k$ th element of the Fibonacci sequence defined by $f_{0}=0, f_{1}=1, f_{k+1}=f_{k}+f_{k-1}$. for $k \geq 1$. Thus the Petrie polygon is

$$
\frac{1}{0}, \frac{0}{1}, \frac{1}{1}, \frac{1}{2}, \frac{2}{3}, \frac{3}{5} \cdots
$$

Lemma 4.1. The matrix $P=\left(\begin{array}{ll}0 & 1 \\ 1 & 1\end{array}\right)$ maps each vertex of the Petrie polygon of $\mathcal{M}_{3}$ to the next one and also $P^{k}=\left(\begin{array}{cc}f_{k-1} & f_{k} \\ f_{k} & f_{k+1}\end{array}\right)$

The proof follows immediately from the definition of the Fibonacci sequence, and induction.

Note that $P$ having determinant -1 is not an element of $\Gamma$ but $T=P^{2}=\left(\begin{array}{ll}1 & 1 \\ 1 & 2\end{array}\right)$ is an element of $\Gamma$.

In the following sections we will consider the Petrie polygon modulo $n$. As $\frac{a}{b}=\frac{-a}{-b}$, we introduce the following concept.

Definition 4.2. We call the least positive integer $m$ with the property that $f_{m-1} \equiv \pm 1$, $\bmod n, f_{m} \equiv 0 \bmod n$ the semi-period $\sigma(n)$ of the Fibonacci sequence $\bmod n$. The period $\pi(n)$ is the least positive integer $m$ such that $f_{m-1} \equiv 1 \bmod n, f_{m} \equiv 0 \bmod n$.

For example if $m=7$, the Fibonacci sequence mod 7 is $0,1,1,2,3,5,1,6,0$, so that $\sigma(7)=8$ and $\pi(7)=16$. The function $\pi$ has been quite well-studied in the literature and is often called the Pisano period. See [8].

\section{The principal congruence subgroups}

The most well-known normal subgroup of the modular group are the principal congruence subgroups. Let $n \in \mathbb{Z}$, Then the principal congruence subgroup of level $n$ in $\Gamma$ is the subgroup

$$
\Gamma(n)=\left\{\left(\begin{array}{ll}
a & b \\
c & d
\end{array}\right) \in \Gamma:\left(\begin{array}{ll}
a & b \\
c & d
\end{array}\right) \equiv \pm\left(\begin{array}{ll}
1 & 0 \\
0 & 1
\end{array}\right) \bmod n\right\}
$$

Now $\Gamma(n)$ is a normal subgroup of $\Gamma$ and so corresponds to a regular map $\mathcal{M}_{3}(n)$ which lies on the surface $\mathbb{H}^{*} / \Gamma(n)$ where $\mathbb{H}^{*}=\mathbb{H} \cup \mathbb{Q} \cup\{\infty\}$.

Another important group for us is $\Gamma_{1}(n)$. This is defined as

$$
\Gamma_{1}(n)=\left\{\left(\begin{array}{ll}
a & b \\
c & d
\end{array}\right) \in \Gamma:\left(\begin{array}{ll}
a & b \\
c & d
\end{array}\right) \equiv \pm\left(\begin{array}{ll}
1 & b \\
0 & 1
\end{array}\right) \bmod n\right\}
$$

where $0 \leq b<n$.

We will not make use of this subgroup but in [2] it was shown that the left cosets of $\Gamma_{1}(n)$ in $\Gamma$ are in one-to-one correspondence with the vertices of $\mathcal{M}_{3}(n)$.

$\Gamma(n)$ is a normal subgroup of $\Gamma$ of index

$$
\frac{n^{3}}{2} \Pi_{p \mid n}\left(1-\frac{1}{p^{2}}\right) \text {. }
$$




\section{The Petrie polygons of $\mathcal{M}_{3}(n)$}

Our principle object of study are the Petrie polygons of the PC-maps $\mathcal{M}_{3}(n)$. We can regard $\mathcal{M}_{3}(n)$ as $\hat{\mathcal{M}}_{3}(3, \infty) / \Gamma(n)$, that is as a quotient of the Farey map. We illustrate our study with the classical regular map $\mathcal{M}_{3}(7)$. This is known as the Klein map and is a map of type $\{3,7\}$. This lies on Klein's Riemann surface of genus 3, known as the Klein quartic. Petrie polygons for this map appear on page 320 in the classic paper [4], although they were not called Petrie polygons there. In fact, Petrie polygons are named after John Flinders Petrie (1907-1972), and Klein's paper [4] was written in 1878. Three of the Petrie polygons are drawn on page 320 of "The Eightfold Way" [5]. The eight in the title comes from the fact that the size of the Petrie polygons is 8 . This will be a special case of results in this paper where we determine the sizes of of the Petrie polygons in PC maps.

In general we observe that the group $\Gamma / \Gamma(n)$ has a transitive action on the Petrie polygons of $\mathcal{M}_{3}(n)$. For $\Gamma$ clearly has a transitive action on the darts of $\mathcal{M}_{3}(\infty)$, and so $\Gamma / \Gamma(n)$ has an induced action on the darts of $\mathcal{M}_{3}(n)$. Clearly, this action will give a transitive action on the set of Petrie polygons of $\mathcal{M}_{3}(n)$.

The vertices of $\mathcal{M}_{3}(n)$ are equivalence classes of vertices of $\hat{\mathcal{M}}_{3}(3, \infty)$. We let $\left[\frac{a}{b}\right]$ denote the equivalence class of $\frac{a}{b}$ in $\mathcal{M}_{3}(n)$ and $\left[\frac{a}{b}\right]$ is joined by an edge to $\left[\frac{c}{d}\right]$ in $\mathcal{M}_{3}(n)$ if and only if $a d-b c \equiv 1 \bmod n$.

The points $\left[\frac{1}{0}\right],\left[\frac{0}{1}\right], \cdots\left[\frac{f_{r-1}}{f_{r}}\right] \cdots$ form the vertices of a Petrie polygon which we call $\operatorname{Pe}(n)$.

Recall the definition of the semiperiod $\sigma(n)$ in section 4 .

Theorem 6.1. The Petrie length of $P e(n)$ is equal to $\sigma(n)$.

Proof. $f_{\sigma(n)-1}=f_{\sigma(n)+1}= \pm 1, f_{\sigma(n)}=0$, so the result follows.

Note that $\operatorname{Pe}(n)$ is a Petrie polygon on $\mathcal{M}_{3}(n)$.

The Fibonacci sequence mod 7 is $0,1,1,2,3,5,1,6,0$ and the Petrie polygon $\mathrm{Pe}(7)$ has vertices $\left[\frac{1}{0}\right],\left[\frac{0}{1}\right],\left[\frac{1}{1}\right],\left[\frac{1}{2}\right]\left[\frac{2}{3}\right],\left[\frac{3}{5}\right],\left[\frac{5}{1}\right],\left[\frac{1}{6}\right]$. The next vertex is $\left[\frac{-1}{0}\right]$ which is equal to $\left[\frac{1}{0}\right]$ so we have closed up our polygon, which has 8 vertices. This polygon is illustrated in Figure 2, where we denote $\left[\frac{a}{b}\right]$ by $(a, b)$. This picture of the Klein surface comes from [2]. The same picture also appears in at the paper [6]. We can apply the same idea for other values of $n$. For example $\mathcal{M}_{5}$ is the icosahedron and $\sigma(5)=10$ which is the known Petrie length for the icosahedron.

\section{The universal Petrie polygon}

To determine the stabiliser of a Petrie polygon it is useful to introduce a new idea. We first extend the standard Fibonacci sequence to include negative numbers. We still want the basic recurrence relation $f_{t-1}+f_{t}=f_{t+1}$ to hold, so this extended Fibonacci sequence is

$$
\cdots-3,2,-1,1,0,1,1,2,3, \cdots
$$

so that $f_{-k}=(-1)^{k-1} f_{k}$. The universal Petrie polygon $\operatorname{Pe}(\infty)$ is the infinite polygon with vertices $\frac{f_{k}}{f_{k+1}}$ where $k \in \mathbb{Z}$ and edges the closed intervals $\left[\frac{f_{k-1}}{f_{k}}, \frac{f_{k}}{f_{k+1}}\right]$. Note that $\frac{f_{-k}}{f_{-k+1}}=\frac{(-1)^{k-1} f_{k}}{(-1)^{k} f_{k-1}}=-\frac{f_{k}}{f_{k-1}}$ and hence the transformation $R: t \mapsto-\frac{1}{t}$ represented by the matrix $\left(\begin{array}{cc}0 & 1 \\ -1 & 0\end{array}\right)$ is an automorphism of $\operatorname{Pe}(\infty)$. Also $T=P^{2}=\left(\begin{array}{ll}1 & 1 \\ 1 & 2\end{array}\right)$ is an 


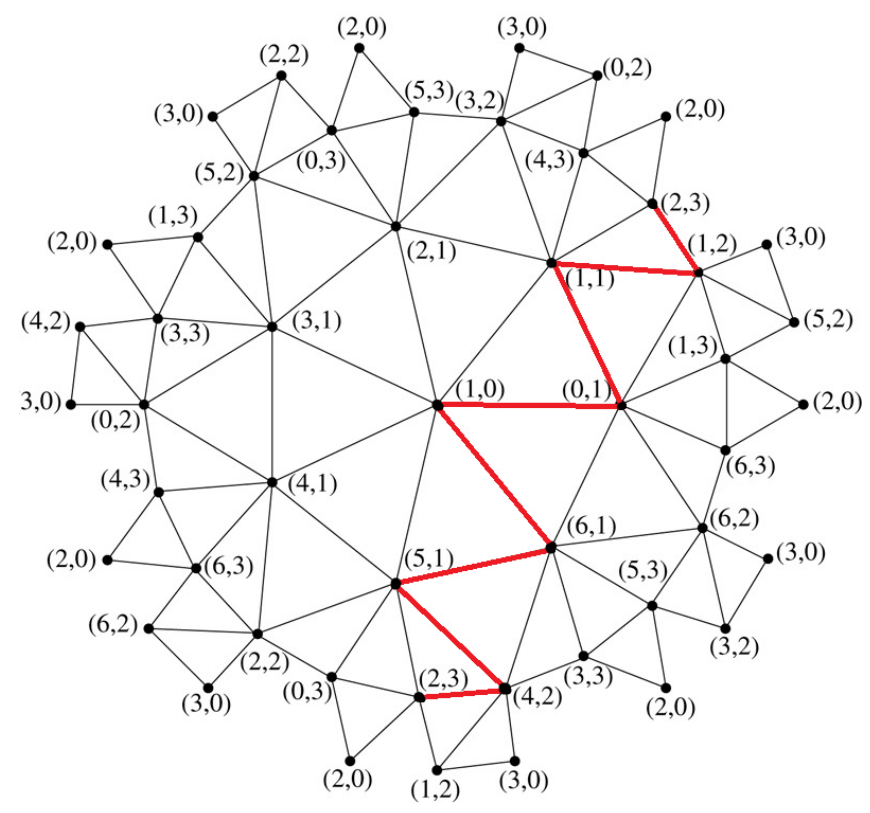

Figure 2: Petrie Polygon

automorphism of $\operatorname{Pe}(\infty)$ that belongs to $\Gamma$. Note that $R^{2}=(T R)^{2}=I$ so that $T$ and $R$ generate an infinite dihedral group of automorphisms of $\operatorname{Pe}(\infty)$.

Theorem 7.1. The automorphism group of $\mathrm{Pe}(\infty)$ in $\Gamma$ is equal to $\langle T, R\rangle \cong D_{\infty}$.

Proof. The group $\langle T, R\rangle$ is a group of automorphisms of $\mathrm{Pe}(\infty)$. We show that it acts transitively on $\mathrm{Pe}(\infty)$. First of all, $P$ maps each Farey fraction two steps along the Farey sequence as

$$
T\left(\frac{f_{k-1}}{f_{k}}\right)=\frac{f_{k-1}+f_{k}}{f_{k-1}+2 f_{k}}=\frac{f_{k+1}}{f_{k+1}+f_{k}}=\frac{f_{k+1}}{f_{k+2}}
$$

Thus the union of the orbits of $\frac{0}{1}$ and $\frac{1}{0}$ under $\langle T\rangle$ is the whole of $\operatorname{Pe}(\infty)$, and as $R\left(\frac{1}{0}\right)=\frac{0}{1}$, $\langle T, R\rangle$ acts transitively on $\operatorname{Pe}(\infty)$. We note that the stabiliser of $\frac{1}{0}=\infty$ in $\operatorname{Aut} \operatorname{Pe}(\infty)$ is trivial. For the stabilizer of $\infty$ in $\Gamma$ consists of the transformations $z \mapsto z+m$, where $m \in \mathbb{Z}$, and the only translation that preserves $\operatorname{Pe}(\infty)$ is the identity, Now suppose that $A \in \operatorname{AutPe}(\infty)$. Then $A(\infty)=\frac{f_{k-1}}{f_{k}}$. By transitivity, there exists $B \in\langle T, R\rangle$ such that $B(\infty)=\frac{f_{k-1}}{f_{k}}$. Thus $A^{-1} B$ fixes $\infty$ and thus $A=B$.

We now search for the automorphism group of $P e(n)$.

Theorem 7.2. The automorphism group of Pe(n) is isomorphic to $D_{\sigma(n) / 2}$. 
Proof. We have an epimorphism $\theta: \Gamma \longrightarrow P S L\left(2, \mathbb{Z}_{n}\right)$ and $\theta(T)=P=\left(\begin{array}{ll}1 & 1 \\ 1 & 2\end{array}\right)$, where we think of this matrix as lying in $\operatorname{PSL}\left(2, \mathbb{Z}_{n}\right)$.

Now $P^{\sigma(n)}=\left(\begin{array}{cc}f_{\sigma(n)-1} & f_{\sigma(n)} \\ \left.f_{\sigma(n)}\right) & f_{\sigma(n)+1}\end{array}\right)$.

Now $f_{\sigma(n)} \equiv 0 \bmod n$ and $f_{\sigma(n)-1}=f_{\sigma(n)+1}= \pm 1$, by the definition of $\sigma(n)$. Thus $P^{\sigma(n)}= \pm I$ and so $T^{\sigma(n) / 2}= \pm I$. which is the identity in PSL $\left(2, \mathbb{Z}_{n}\right)$ Thus the automorphism group of $\operatorname{Pe}(n)$ is generated by $R$ and $T$ with $R^{2}=(R T)^{2}=T^{\sigma(n) / 2}=1$ and hence $\langle R, T\rangle \cong D_{\sigma(n) / 2}$ of order $\sigma(n)$.

It is interesting to see how this theorem works in practice, so let us go back to our example of $n=7$ as illustrated in Figure 2. As $\sigma(7)=8$ we have an action of $D_{4}$ on $\operatorname{Pe}(7)$ an 8-sided polygon. The element $T$ has two cycles of length 4 , namely

$(1,0) \longrightarrow(1,1) \longrightarrow(2,3) \longrightarrow(5,1) \longrightarrow(1,0)$

$(0,1) \longrightarrow(1,2) \longrightarrow(3,5) \longrightarrow(1,6) \longrightarrow(0,1)$

and for the involution $R$ we have $(1,0) \leftrightarrow(0,1),(1,1) \leftrightarrow(6,1),(1,2) \leftrightarrow(5,1),(2,3)$ $\leftrightarrow(4,2)$.

(Note that $\left[\frac{3}{5}\right]=\left[\frac{-4}{-2}\right]=\left[\frac{4}{2}\right]$ so that $(3,5)=(4,2)$, etc.)

As $\Gamma / \Gamma(n)$ acts transitively on the darts of $\mathcal{M}_{3}(n)$ we use equation (1) in section 5 to obtain

Corollary 7.3. The number of Petrie polygons on $\mathcal{M}_{3}(n)$ is equal to

$$
\frac{n^{3}}{2 \sigma(n)} \Pi_{p \mid n}\left(1-\frac{1}{p^{2}}\right) \text {. }
$$

Example. Let $n=7$. Then $\sigma(7)=8$. The number of Petrie polygons of $\mathcal{M}_{3}(7)$ is equal to 21 . Klein drew three of them in [5]. The others can be found by rotating these through $2 \pi k / 7$, for $k=1, \cdots 6$.

\section{More about $\sigma(n)$}

Theorem 8.1. For all positive integers $m>2, \sigma(m)$ is even.

Proof. $P^{\sigma(m)}=\left(\begin{array}{cc}f_{\sigma(m)-1} & f_{\sigma(m)} \\ f_{\sigma(m)} & f_{\sigma(m)+1}\end{array}\right) \equiv \pm 1 \bmod m$

Thus $(\operatorname{det} P)^{\sigma(m)} \equiv 1 \bmod m$, so $(-1)^{\sigma(m)} \equiv 1 \bmod m$ and thus $\sigma(m)$ is even.

Exactly the same proof shows that $\pi(m)$ is even for $m>2$. A much easier proof than that given in [8].

Let $\rho=\frac{1+\sqrt{5}}{2}$ (the golden ratio) and $\rho^{*}=\frac{1-\sqrt{5}}{2}$. Note that $\rho \rho^{*}=-1$ and $\rho+\rho^{*}=1$.

Let $\mathbb{Z}_{n}[\rho]=\{a+b \rho \mid a, b \in \mathbb{Z} /(n)\}$ and if $\alpha=a+b \rho$, define $\alpha^{*}=a+b \rho^{*}$. Then $(\alpha \beta)^{*}=\alpha^{*} \beta^{*}$.

We define the norm $N$ on $\mathbb{Z}_{n}[\rho]$ by $N(\alpha)=\alpha \alpha^{*}$. Then $N(\alpha \beta)=N(\alpha) N(\beta)$. We call $\alpha$ a unit if $N(\alpha)= \pm 1$, so that $\rho$ is a unit. The units of $\mathbb{Z}_{n}[\rho]$ form a group $\mathbb{Z}_{n}^{*}[\rho]$ under multiplication.

Theorem 8.2. $\sigma(n)$ is the order of $\rho$ in $\mathbb{Z}_{n}^{*}[\rho]$, if $f_{\sigma(n)-1}=1$ and is equal to half the order of $\rho$ if $f_{\sigma(n)-1}=-1$. In all cases $\pi(n)$ is equal to the order of $\rho$ in $\mathbb{Z}_{n}^{*}[\rho]$. 
Proof. From $\rho^{2}=\rho+1$ we can use induction to prove that $\rho^{m}=f_{m} \rho+f_{m-1}$ Thus if $m=\sigma(n), f_{m}=0$ and $f_{m-1}= \pm 1$. Thus $\rho^{\sigma(n)}=1$ if $f_{\sigma(n)-1}=1$ and is equal to -1 if $f_{\sigma(n)-1}=-1$ and in the latter case the order of $\rho$ is $2 n$.

The proof for $\pi$ is similar.

\section{The Pisano period and the semiperiod}

There is a lot about the Pisano period $\pi(m)$ in the literature . For example, see [8], where the Pisano period is calculated for all primes less than 2000. Very little is known about the semiperiod $\sigma(m)$. We end with a few results comparing the two.

Lemma 9.1. $\pi(m)=\sigma(m)$ if and only if $f_{\sigma(m)-1}=1 . \pi(m)=2 \sigma(m)$ if and only if $f_{\sigma(m)-1}=-1$.

Proof. Let $k$ be the least integer such that, modulo $m, f_{k-1}=-1, f_{k}=0$. Then $k=\sigma(m)$ and $f_{\sigma(m)+r}=-f_{\sigma(r)}$, so that $f_{2 \sigma(m)-1}=-f_{\sigma(m)-1}=1, f_{2 \sigma(m)}=0$ and $\pi(m)=$ $2 \sigma(m)$.

Alternatively, $f_{k-1}=+1$ and then $\pi(m)=\sigma(m)$.

We want to determine which of these occur. We give some partial answers. From Theorem 9.2 we see that $\sigma(m)=\pi(m)$ if and only if $\rho^{m-1}=1$ and $\sigma(m)=2 \pi(m)$ if and only if $\rho^{m-1}=+1$.

Theorem 9.2. Let $p \equiv \pm 2 \bmod 5$ be an odd prime. Then $\pi(p)=2 \sigma(p)$.

Proof. The point is that 5 is a quadratic residue $\bmod p$ if and only if $p \equiv \pm 1 \bmod 5$. Otherwise 5 is a non-residue and by adjoining $\sqrt{5}$ to $F_{p}$, the finite field of $p$ elements which we can take to be $\mathbb{Z} / p \mathbb{Z}$, we get a finite field $K$ of characteristic $p$ with $p^{2}$ elements. This field can be considered to be $F_{p} / I$, Where $I$ is the ideal generated by $x^{2}-x-1$, which we can identify with all elements of the form $a+b \rho$, where $a, b \in F_{p}$ the field with $p$ elements. The polynomial $x^{2}-x-1$ has no roots in $F_{p}$ but two roots in $K$ interchanged by the Frobenius automorphism $\phi: a \longrightarrow a^{p}$. If $\alpha$ is a root of this polynomial then the other root is $a^{p}=1-\alpha$ and hence so that $a^{p+1}=\alpha-\alpha^{2}=-1$. Thus, by Theorem 8.2 and Lemma 9.1, $\pi(p)=2 \sigma(p)$.

Theorem 9.3. Let $p \equiv 11,19 \bmod 20$. Then $\pi(p)=\sigma(p)$.

Proof. We have $p \equiv \pm 1 \bmod 5$ and so 5 has a square root in $F_{p}$ the finite field with $p$ elements and hence $\rho \in F_{p}$. Its multiplicative group has order $p-1$. Now

$$
\rho^{\pi(p)}=f_{\pi(p)} \rho+f_{\pi(p)-1} \equiv 1 \bmod p
$$

Therefore $\pi(p)$ is a divisor of $p-1$. Now $p \equiv 3 \bmod 4$ so that $p=4 k+3$ for some integer $k$. This $p-1=4 k+2$. If $\pi(p)=2 \sigma(p)$, then $\sigma(p)$ is a divisor of $2 k+1$ and thus $\sigma(p)$ is odd contradicting Theorem 8.1. Therefore $\sigma(p)=\pi(p)$.

We would like to thank Tom Harris for helping us with the results in section 9 and the referee for his careful reading of the manuscript. 


\section{References}

[1] H. S. M. Coxeter and W. O. J. Moser, Generators and relations for discrete groups, volume 14 of Ergebnisse der Mathematik und ihrer Grenzgebiete [Results in Mathematics and Related Areas], Springer-Verlag, Berlin-New York, 4th edition, 1980.

[2] I. Ivrissimtzis and D. Singerman, Regular maps and principal congruence subgroups of Hecke groups, European J. Combin. 26 (2005), 437-456, doi:10.1016/j.ejc.2004.01.010.

[3] G. A. Jones and D. Singerman, Theory of maps on orientable surfaces, Proc. London Math. Soc. (3) 37 (1978), 273-307.

[4] F. Klein, Ueber die Transformation siebenter Ordnung der elliptischen Functionen, Math. Ann. 14 (1878), 428-471, doi:10.1007/BF01677143.

[5] S. Levy, The eightfold way: the beauty of Klein's quartic curve, volume 35, Cambridge University Press, 1999.

[6] E. Schulte and J. M. Wills, A polyhedral realization of Felix Klein's map $\{3,7\}_{8}$ on a Riemann surface of genus 3, J. London Math. Soc. (2) 32 (1985), 539-547, doi:10.1112/jlms/s2-32.3.539.

[7] D. Singerman, Universal tessellations, Rev. Mat. Univ. Complut. Madrid 1 (1988), 111-123.

[8] D. D. Wall, Fibonacci series modulo m, Amer. Math. Monthly 67 (1960), 525-532. 